\title{
CLASSIFICAÇÃO DE GERMOPLASMA DE Dioscorea sp. ATRAVÉS DA ANÁLISE DAS COMPONENTES PRINCIPAIS
}

\author{
CLASSIFICATION OF Dioscorea sp. GERMPLASM BY \\ PRINCIPAL COMPONENTS ANALISYS
}

\author{
Péricles de Albuquerque Melo Filho ${ }^{1}$ Roseane Cavalcanti dos Santos ${ }^{2}$ \\ Rivaldo Chagas Mafra José Weilinghton dos Santos ${ }^{2}$ \\ Clodoaldo José da Anunciação Filho ${ }^{1}$
}

RESUMO

Procedeu-se a classificação interespecífica em 11 acessos de cará, pertencentes à Coleçâo de Germoplasma da Universidade Federal Rural de Pernambuco, (UFRPE), com objetivo de caracterizá-los morfologicamente e classificá-los com base nos estudos dos componentes principais. Quatorze descritores foram tomados de cada acesso para a referida análise: cor e forma da folha, pigmentação do pecíolo, cor e forma da haste, presença e cor de membranas, presença de acúleos, presença de túberas aéreas, forma e pigmentação da túbera, presença de raízes nas túberas, comprimento e largura da folha. Entre os descritores analisados, observou-se que o comprimento e a largura da folha foram os de maior contribuição na discriminação dos acessos. A porcentagem de variância acumulada para a $1^{\circ}$ e $2^{\circ}$ componentes principais foi de $89 \%$ da variação total. Na representação gráfica dos acessos, observouse a formação de seis grupos representando os acessos de Dioscorea sp.

Palavras-chave: cará, análise multivariada, descritores.

\section{SUMMARY}

Interspecific classification $m$ Dioscorea germplasm was made aiming to characterize them by morphological descriptors and classify by principal component analysis. Eleven accesses belonging to Universidade Federal Rural de Pernambuco Brasil (UFRPE Germplasm Collection) were studied. Fourteen descriptors were chosen for each access: leaf color and shape, petiole pigment, stem color and shape, presence and color of membranes, presence of good, presence of aerial tubers, tuber shape, presence of roots $m$ lhe tuber, pigment in the tuber and leaf length and width. It was observed that leaf length and width provided high contribution on the access discriminations. The accumulated variance to the $1^{\text {st }}$ and $2^{\text {nd }}$ principal components were $89 \%$ of the whole variation. Six groups were made up representing Dioscorea accesses.

Key words: yam, multivatiate analysis, descriptors.

\section{INTRODUÇÃO}

No melhoramento de plantas, três recursos básicos devem ser explorados para que se consiga maior variabilidade genética. Primeiro, é o conhecimento das diferenças hereditárias entre os acessos da espécie cultivada; o segundo, refere-se às diferenças que podem ser criadas artificialmente pelo uso de mutagênicos, e o terceiro, são as diferenças que ocorrem entre os relativos selvagens das espécies cultivadas (FARIAS, 1996). Segundo JATASRA \& PARODA (1983), a informação precisa sobre a divergência genética é decisiva para o sucesso de um programa de melhoramento, uma vez que plantas geneticamente divergentes produzem alto efeito heterótico e, conseqüentemente, segregantes desejáveis para os propósitos do programa. Em bancos de germoplasma, este estudo pode ser realizado através da utilização de vários descritores, desde os fitotécnicos até os moleculares. Para se quantificar e descrever a variação em termos de um número reduzido de descritores, os métodos multivariados têm sido os mais utilizados pelos melhoristas, e a análise dos componentes principais é empregada

\footnotetext{
${ }^{1}$ Professor, Engenheiro Agrônomo, MSc., Universidade Federal Rural de Pernambuco (UFRPE)-DEPA, Rua: Don Manoel de Medeiros s/n, Dois Irmãos, 50171-900 ,Recife- PE. E-mail: pericles@unb.br. Autor para correspondência.

${ }^{2}$ Engenheiro Agrônomo, MSc., Pesquisador Embrapa Algodão, Campina Grande - PB.
} 
com frequência, uma vez que condensa a variância de um conjunto de dados em poucos eixos, tomando possível a análise de maior parte dessa variância em duas ou três dimensões ou componentes.

A Universidade Federal Rural de Pernambuco (UFRPE) possui, há mais de 30 anos, uma Coleção de Germoplasma de Dioscorea, composto de três espécies distribuídas em 11 acessos. Embora seja relativamente pequena, a diversidade fenotípica detectada nos acessos tem sido suficiente para gerar variabilidade genética para ser explorada em programas de melhoramento.

O género Dioscorea é composto de mais de 600 espécies largamente distribuídas pelas regiões temperadas e tropicais do mundo. $\mathrm{O}$ número básico de cromossomos no género é $\mathrm{n}=10$. As séries poliplóides são compostas de auto e alopoliplóides. De acordo com Santos (1996), a maior parte das espécies cultivadas são oriundas da Ásia e do Oeste da África, sendo as de maior importância económica $D$. cayanensis e $D$. rotundata oriundas do Oeste da África, D. alata, originária da Ásia e D. trifida, nativa da América tropical (Purseglove, 1975). A espécie D. alata é cultivada em larga escala para fins comestíveis; adapta-se bem a situações agroecológicas de alta temperatura, elevada pluviosidade e fotoperíodo curto.

$\mathrm{Na}$ região Nordeste, as espécies mais cultivadas são $\boldsymbol{D}$. alata, representada pela variedade São Tomé e D. cayanensis, com a variedade Da Costa, cultivada em escala nacional. Geralmente, as cultivares da espécie alata são muito produtivas. A estimativa de rendimento situa-se entre 10 a $15 \mathrm{~kg}$ de túberas/planta/ano e algumas têm a característica de possuir túberas aéreas (MARTIN, 1972); os frutos, contudo, ocasionalmente se desenvolvem e quando o fazem, ou não possuem sementes, ou essas são pequenas e chochas, inviabilizando sua propagação. Segundo esse autor, os grãos de pólen dessa espécie raramente germinam tanto em ambiente in vivo quanto in vivo.

Neste trabalho, procedeu-se a classificação interespecífíca nos onze acessos de Dioscorea da Coleção da UFRPE, com objetivo de caracterizá-los morfologicamente e classificá-los quanto à divergência com base nos estudos dos componentes principais.

\section{MATERIAL E MÉTODOS}

A pesquisa foi realizada em campo, no período de março a setembro de 1998, na área experimental do setor da Fitotecnia da UFRPE, em Recife, PE. Os onze acessos da coleção de cará foram plantados no espaçamento de $1,00 \times 0,60 \mathrm{~m}$, em fileiras individuais, de $10 \mathrm{~m}$ de comprimento, constituindo a parcela experimental com densidade de duas plantas por metro linear. O solo onde foi cultivado os acessos é do tipo Podzol hidromórfico, de textura franco arenosa e ácido. A adubação de manutenção foi baseada na análise de fertilidade do solo. Aplicaram-se, na área, $60 \mathrm{~kg} / \mathrm{ha}$ de sulfato de amónio em cobertura (metade aos 60 dias após o plantio e o restante aos 90$), 90 \mathrm{~kg} / \mathrm{ha}$ de superfosfato simples e $40 \mathrm{~kg} / \mathrm{ha}$ de cloreto de potássio, ambos no plantio, em fundação. Durante a condução do experimento, as médias de temperatura noturna e diurna registradas no campo foram, respectivamente $20^{\circ} \mathrm{C}$ e $29^{\circ} \mathrm{C}$; a média da umidade relativa do ar foi $88 \%$. O volume total registrado para a precipitação pluvial foi igual a $1.680 \mathrm{~mm}$.

Foram utilizados para o presente estudo quatorze descritores morfológicos, cujas medidas de amostragens foram seguidas de acordo com metodologias descritas por RHODES \& MARTIN (1972) e pelo IPGRI/IITA (1997). Os descritores foram os seguintes: cor $\mathrm{e}$ forma $\mathrm{da}$ folha, pigmentação do pecíolo, cor e forma da haste, presença e cor da membrana, presença de acúleos e de túberas aéreas, forma e pigmentação da túbera, presença de raízes, comprimento e largura da folha. Cada descritor foi registrado tomando-se como base uma amostra ao acaso de $50 \%$ das plantas dentro da parcela. Todos os descritores vegetativos foram registrados aos 40 dias após a brotação (dab); os relativos às túberas, foram registrados aos 210 dab. A caracterização morfológica dos acessos encontrase na tabela 1 .

A classificação dos acessos foi procedida através da análise das componentes principais calculadas a partir da matriz de variâncias e covariâncias segundo metodologia descrita por MOREIRA et al. (1994).

\section{RESULTADOS E DISCUSSÃO}

Os autovetores e as porcentagens da variância acumulada para a primeira e segunda componentes principais encontram-se na tabela 2 . Observa-se que, para a primeira componente principal, os descritores de maior contribuição na discriminação dos acessos foram comprimento da folha e largura da folha; na segunda componente, destacaram-se, além desses já citados, as formas da túbera e da folha. Esses, portanto, seriam os caracteres morfológicos mais responsivos nos processos seletivos entre acessos do género Dioscorea. A porcentagem da variância acumulada para o $1^{\mathrm{a}}$ e $2^{\mathrm{a}}$ componentes principais acumulou $89 \%$ da variação total. 
Tabela 1 - Descritores morfológicos dos onze acessos de Dioscorea sp. Recife, 1998.

\begin{tabular}{|c|c|c|c|c|c|c|c|c|c|c|c|c|c|c|c|c|}
\hline Acesso & Nome & Origem & $\mathrm{CF}$ & $\mathrm{FF}$ & PP & $\mathrm{CH}$ & $\mathrm{FH}$ & PM & $\mathrm{CM}$ & PA & PTA & FT & PR & PT & $\mathrm{CPF}$ & LF \\
\hline UFRPE 01 & Da Costa ${ }^{a}$ & PE & 1 & 1 & 2 & 1 & 1 & 2 & 1 & 1 & 2 & 1 & 2 & 2 & 3 & 2 \\
\hline UFRPE 02 & Florida ${ }^{b}$ & SP & 2 & 1 & 2 & 1 & 2 & 1 & 1 & 2 & 2 & 1 & 1 & 2 & 5 & 1 \\
\hline UFRPE 03 & $\mathrm{Nambu}^{\mathrm{b}}$ & $\mathrm{PE}$ & 1 & 2 & 2 & 1 & 2 & 1 & 1 & 2 & 2 & 2 & 1 & 2 & 9 & 6 \\
\hline UFRPE 04 & Mandioca $^{b}$ & PE & 1 & 1 & 2 & 2 & 2 & 1 & 2 & 2 & 2 & 1 & 1 & 2 & 10 & 5 \\
\hline UFRPE 05 & Ilhéus ${ }^{\mathrm{b}}$ & BA & 3 & 1 & 1 & 2 & 2 & 1 & 2 & 2 & 2 & 1 & 1 & 1 & 10 & 6 \\
\hline UFRPE 06 & Sorocaba $^{\mathrm{b}}$ & SP & 1 & 1 & 2 & 1 & 2 & 1 & 1 & 2 & 2 & 1 & 1 & 2 & 5 & 3 \\
\hline UFRPE 07 & Ceilão" & SP & 3 & 1 & 1 & 2 & 2 & 1 & 2 & 2 & 2 & 3 & 2 & 2 & 1 & 1 \\
\hline UFRPE 08 & $\mathrm{IAC}^{\mathrm{b}}$ & SP & 2 & 1 & 2 & 1 & 2 & 1 & 1 & 2 & 2 & 3 & 1 & 2 & 6 & 4 \\
\hline UFRPE 09 & Cole do Mato ${ }^{\mathrm{b}}$ & $\mathrm{PE}$ & 2 & 1 & 2 & 1 & 2 & 1 & 1 & 2 & 2 & 2 & 1 & 1 & 2 & 1 \\
\hline UFRPE 10 & Cará Moela $^{c}$ & PE & 1 & 3 & 2 & 1 & 1 & 2 & 1 & 2 & 1 & 3 & 1 & 2 & 6 & 8 \\
\hline UFRPE 11 & São Toméb & $\mathrm{PE}$ & 1 & 1 & 2 & 1 & 2 & 1 & 1 & 2 & 2 & 3 & 1 & 2 & 10 & 6 \\
\hline
\end{tabular}

Legenda: CF- cor da folha: 1 - verde, 2 - verde escura, 3 - arroxeada; FF - forma da folha: 1 - sagitada, 2 - lobada, 3 - cordiforme; PP - pigmentação do pecíolo: 1 - presente, 2 - ausente; $\mathrm{CH}$ - cor da haste: 1 - verde, 2 - verde-arroxeada; FH - forma da haste: 1 - cilíndrica, 2 - quadrangular; PM - presença de membrana: 1 - presente, 2 - ausente; CM - cor da membrana: 1 - verde, 2 - roxa; PA- presença de acúleos: 1 - presente, 2 - ausente; PTA - presença de túberas aéreas: 1 - presente, 2 - ausente; FT - forma da túbera: 1 - cilíndrica, 2 - esférica, 3 - oval; PR - presença de raízes nas túberas; 1 - presente, 2 - ausente; PT - pigmentação na túbera: 1 - presente, 2 ausente; CPF - comprimento da folha: 1-91-94mm, 2-95-100mm, 3-101-104mm, 4 - 105-110mm, 5-111-114mm, 6 - 115120mm, 7 - 121-124mm, 8 - 125-130 mm, 9 - 131-134mm, 10 - 135-140mm; LF- largura da folha: 1 - 51-54 mm, 2 - 55-60 mm, 3 -61-64 mm, 4 - 65-70mm, 5 - 71-74mm, 6-75-80mm, 7 -81-84mm, 8-85-90mm. ${ }^{a}-$ D. cayanensis; ${ }^{b}$-D. alata; ${ }^{c}-$ D. Bulbiforme.

$\mathrm{Na}$ figura 1, encontra-se a representação gráfica dos acessos no sistema de eixos, representando a primeira e a segunda componentes principais. Baseados na dispersão espacial dos acessos no plano multidimensional e nas suas

Tabela 2 - Autovetores e porcentagens da variância acumulada para a primeira $(\mathrm{C} 1)$ e segunda $(\mathrm{C} 2)$ componentes principais para os descritores morfológicos de Dioscorea sp.

\begin{tabular}{|c|c|c|}
\hline \multirow[t]{2}{*}{ Descritores } & \multicolumn{2}{|c|}{ Componente principal } \\
\hline & primeira & segunda \\
\hline Cor da folha & -0.07 & -0.13 \\
\hline Forma da folha & 0.05 & 0.32 \\
\hline Pigmentação do pecíolo & 0.01 & 0.03 \\
\hline Cor da haste & 0.01 & -0.10 \\
\hline Forma da haste & -0.01 & -0.17 \\
\hline Presença de membrana & 0.01 & 0.17 \\
\hline Cor da membrana & 0.02 & -0.10 \\
\hline Presença de acúleos & -0.01 & -0.01 \\
\hline Presença de túberas aéreas & 0.01 & -0.16 \\
\hline Forma da túbera & 0.01 & 0.37 \\
\hline Presença de raízes nas túberas & -0.06 & 0.03 \\
\hline Pigmentação na túbera & -0.02 & 0.05 \\
\hline Comprimento da folha & 0.81 & -0.47 \\
\hline Largura da folha & 0.57 & 0.64 \\
\hline Variância acumulada (\%) & 76,10 & 89,12 \\
\hline
\end{tabular}

características seis grupos, quatro dos quais constituídos de apenas um acesso. Observa-se nessa figura que Cará Moela (D. bulbiforme) (Grupo l) foi o acesso que se manteve mais afastado dos demais estudados. Suas características mais marcantes foram o aspecto exclusivo de suas folhas, únicas com formato cordiforme, e a presença de túberas aéreas (Tabela 1). No caso do acesso Da Costa (D. cayanensis) (Grupo 2), que também formou um grupo isolado, observa-se na tabela 1 que esse, junto com o Cará Moela, apresenta caule com formato cilíndrico sem presença de membranas. Sua diferenciação mais acentuada, contudo, se fez perceber através da presença exclusiva de acúleos. Segundo WAITT (1963), essa espécie apresenta outra característica que é a ocorrência de formas tetra e hexaplóide; as flores, quando ocorrem, são férteis e os cruzamentos podem ser realizados com boas perspectivas de plântulas viáveis (WAITT, 1963).

Entre os acessos de $\boldsymbol{D}$. alata, verificou-se que Nambu e Ceilão, formaram grupos separados entre os demais da espécie (Grupos 3 e 4, respectivamente). As diferenças entre Ceilão e os demais acessos concentraram-se, basicamente, na presença de raízes nas túberas e no pequeno comprimento da folha (Tabela 1). Com relação a Nambu, o que o tornou distinto entre os acessos do grupo 5 (Mandioca, Ilhéus e São Tomé) foi o formato da folha, única de aspecto lobado e a forma 


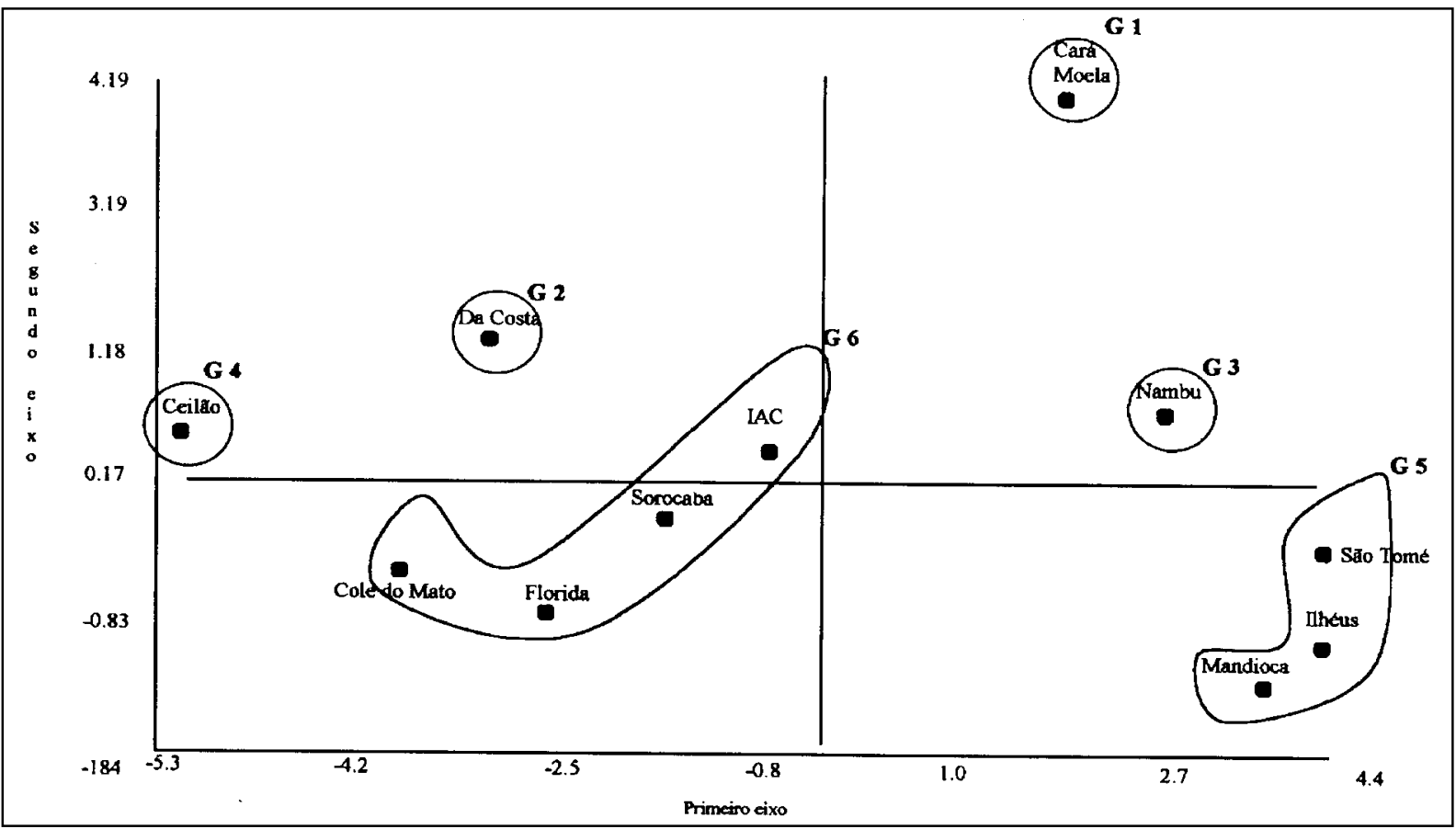

Figura 1 - Distribuição dos pontos correspondentes aos acessos de Discorea sp. no plano definido pelas duas primeiras componentes principais.

esférica das túberas, que além de pequenas pesam cerca de quatro vezes menos, quando comparadas à média entre os alata da coleção (Mafra, 1978).

Nos acessos do grupo 6, a similaridade intra-específíca entre eles foi maior, coincidindo no mínimo em oito caracteres, cuja maior conjunção foi verificada entre Florida e Sorocaba que diferiram apenas na cor da folha e largura do folíolo (Tabela 1). A grande similaridade detectada entre esses dois acessos pode ser devido a ambos tratarem do mesmo germoplasma. A pequena variação morfológica detectada entre eles pode ser oriunda de alguma variação ambiental, no caso da largura do folíolo, ou de uma pequena mutação sofrida em descendentes da planta matriz, que variou para a cor do folíolo. As partes vegetativas, a propósito, variam na quantidade e distribuição de antocianina, cuja expressão desse pigmento depende muito das condições climáticas onde o acesso está sendo desenvolvido. WAITT (1963) enfatiza quealgumas modificações morfoagronômicas que ocorrem em espécies de $\boldsymbol{D}$. alata são devido a efeitos ambientais, mais especificamente relacionadas aos efeitos da temperatura e do fotoperíodo.

\section{CONCLUSÃO}

Os onze acessos da coleção de Dioscorea da UFRPE são discriminados em seis grupos, entre os quais. Cará Moela, Da Costa, Nambu e Ceilão são distintos entre si, de acordo com suas peculiaridades morfológicas e dispersão espacial revelada através da análise das componentes principais.

\section{REFERÊNCIAS BIBLIOGRÁFICAS}

FARIAS, R.H. de. Classificação de germoplasma de amendoim (Arachis hypogaea L.) na base dos componentes principais. Areia : UFPB, 1996. 60p. (Trabalho de graduação).

IPGRI/IITA. Descriptors for yam (Dioscorea sp.). Rome, Italy: Intemational hstitute of Tropical Agriculture, Ibadan, Nigeria/Intemational Plant Genetic Resources Instutrute, 1977.61p.

JATASRA, D.S. PARODA, R.S. Genetic divergence in wheat. Indian Journal of Genetic, índia, n.43, p.63-67,1983.

MAFRA, R.C. Contribuição ao estudo da cultura do cará. Recife : Universidade Federal Rural de Pernambuco, 1978. 20p.

MARTIN, F.W. Yam production methods Washinghton : USDA Agricultural Research Service Production Research, 1972. 72p. Report. n. 147. 
MOREIRA, J. de A.N., SANTOS, J.W. dos, OLIVEIRA, S.R. de variations in yams (Discorea alata L.). Journal of American M. Abordagens e metodologias para avaliação de Society Horticultural Science, New York. v.97, n.5, p.685germoplasma. EMBRAPA/CNPA; Brasília : EMBRAPA688, 1972. SPI, 1994. 115p.

PURSEGLOVE, J.W. Tropical crops monocotyledons. 2 ed. London : Longman, 1975. 607p.

RHODES, A.M., MARTIN, F.W., Multivariate studies of variations in yams (Discorea alata L.). Journal of American
Society Horticultural Science, New York. v.97, n.5, p.685688, 1972.

SANTOS, E.S. Inhame (Dioscorea spp.) - Aspectos básicos da PURSEGLOVE, J.W. Tropical crops monocotyledons. 2 ed. Cultura. João Pessoa : EMEPA-PB, SEBRAE, 1996. 158p. London : Longman, 1975. 607p.

WAITT, A. W. Yains. Diacorea species. Field Crop Abstracts, RHODES, A.M., MARTIN, F. W. Multivariate studies of New York. v.16,n.3,p.145-153, 1963.

Ciência Rural, v. 30, n. 4, 2000. 